\title{
Surveillance culture and Gram staining for detecting the causative bacteria of ventilator-associated pneumonia in neonates
}

Yoshiki Uemura ( $\sim$ geisha3my@yahoo.co.jp )

Takatsuki General Hospital

Masahiro Enomoto

Takatsuki General Hospital

Mio Sakuma

Hyogo College of Medicine

Shin Kikuchi

Takatsuki General Hospital

Atsuko Takei

Takatsuki General Hospital

Hitoshi Ikegami

Takatsuki General Hospital

Hirotaka Minami

Takatsuki General Hospital

Yoshinori Katayama

Takatsuki General Hospital

\section{Research Article}

Keywords: ventilator-associated pneumonia, Gram staining, surveillance culture, neonate

Posted Date: January 5th, 2021

DOI: https://doi.org/10.21203/rs.3.rs-135951/v1

License: (c) (i) This work is licensed under a Creative Commons Attribution 4.0 International License.

Read Full License 


\section{Abstract \\ Background}

To investigate the efficacy of surveillance culture for the rapid detection of pathogens associated with ventilator-associated pneumonia (VAP) in neonates.

\section{Methods}

A retrospective study was conducted on all patients with VAP in the neonatal intensive care unit. Whether causative bacteria on the culture of tracheal aspirates obtained at the onset of VAP matched with those on Gram staining and weekly surveillance culture was investigated.

\section{Result}

The concordance rate between surveillance culture using samples collected 4-10 days before VAP and causative bacteria was $53 \%$. When Gram staining was performed at the onset of VAP, the concordance rate significantly increased to $86 \%(p=0.022)$.

\section{Conclusions}

The concurrent use of surveillance culture and Gram staining for the detection of causative bacteria may be effective in determining initial treatment among neonates with VAP.

\section{Background}

Ventilator-associated pneumonia (VAP) is a common healthcare-related infection in the neonatal intensive care unit (NICU). Moreover, it is associated with worsening symptoms, increased use of antibiotics, high hospitalization cost, long stay in the NICU, and high rate of mortality [1]. In adults, surveillance culture of endotracheal aspirate can identify causative bacteria [2]; however, the use of this method in neonates has not been reported.

Our previous study showed that Gram staining had a high accuracy in diagnosing VAP [3]. However, it cannot always identify causative pathogens, thereby posing challenges in selecting an appropriate antibiotic therapy. Thus, the current study aimed to investigate the efficacy of surveillance culture for the rapid detection of pathogens correlated with VAP. We hypothesized that the combined use of surveillance culture and Gram staining is effective in identifying the causative pathogens of VAP.

\section{Methods}




\section{Setting and Patients}

We retrospectively reviewed cases of VAP that developed in the NICU of Takatsuki General Hospital (Osaka, Japan) between July 2013 and May 2015. This institution has a level III NICU, with a 21-bed capacity. In total, there were 34 VAP cases among 23 neonates. All these cases were included in the analysis.

\section{Sputum studies}

VAP was diagnosed according to the criteria reported in 2009, which was based on Gram staining of tracheal aspirates and clinical symptoms (Katayama et al. 2009) [3]. Briefly, VAP was defined as healthcare-associated pneumonia that develops $>48 \mathrm{~h}$ after starting mechanical ventilation. Patients were diagnosed with VAP when there was an increase in ventilator demand along with higher amounts of endotracheal aspirates and when microorganisms and polymorphonuclear leukocytes were identified on gram-stained smears of aspirates. The diagnosis of VAP was confirmed based on increased levels of serum C-reactive protein, presence of intracellular bacteria on gram-stained smears, or both. The etiological diagnosis of VAP was considered definitive when any microorganism was isolated from tracheal aspirates at concentrations of $\geq 10^{3} \mathrm{cfu} \mathrm{mL}^{-1}$ [4]. To obtain a diagnosis, after performing Gram staining using tracheal aspirates collected at the onset of VAP, we sent the tracheal aspirate samples to the laboratory for the identification of causative bacteria.

During the study period, surveillance culture of nasal cavity (NC) and tracheal aspirate (TA) samples was performed weekly in all neonates who were on ventilator support. Nurses collected TA samples via endotracheal aspiration, and these samples were placed in sterile containers (Nippon Sherwood Medical Industries Ltd., Tokyo, Japan) using a closed aspiration system (Trach Care; Ballard Medical Products, Draper, UT, the USA), and NC samples were cultured using a nasal sterile swab (Seed swaby No. 2; Eiken Chemical Co., Ltd., Tokyo, Japan). NC and TA samples collected 1-7 days before the onset of VAP were referred as NC $1 \mathrm{wk}$ and TA $1 \mathrm{wk}$, respectively, and those collected 8-14 days before the onset as NC 2 wks and TA 2 wks, respectively. The surveillance culture results were available 4 days after the collection of samples at our hospital because the clinical reference can be identified thereafter. Therefore, NC and TA samples collected 4-10 days before the onset of VAP were referred as referable NC and TA, respectively.

\section{Statistical methods}

The Statistical Package for the Social Sciences software (version 24; IBM-SPSS Inc., Armonk, NY) was used for analysis. The Fisher's exact test was utilized to compare concordance between surveillance culture and causative bacteria. $P$ values $<.05$ were statistically significant. This study was approved by the Ethics Committee of Takatsuki General Hospital. (Approval Number: 2016-51). Informed consent was obtained from the patient's parent.

\section{Results}


All VAP cases occurred in neonates born earlier than 28 weeks (Table 1). The median age at VAP onset was 29.5 (range: 9-73) days, and the median duration of endotracheal incubation until VAP onset was 29 (range: 3-69) days (Table 2).

Table 1

Characteristics of patients $(n=23)$

\begin{tabular}{|lll|}
\hline Variables & & \\
\hline Gestational age, wk, median (range) & 24 & $(22-28)$ \\
\hline Birth weight, g, median (range) & 608 & $(424-1058)$ \\
\hline Male gender, $\mathrm{n}(\%)$ & 12 & $(52)$ \\
\hline Vaginal delivery, $\mathrm{n}(\%)$ & 4 & $(17)$ \\
\hline Inborn, $\mathrm{n}(\%)$ & 20 & $(87)$ \\
\hline Antenatal corticosteroid, $\mathrm{n}(\%)$ & 18 & $(78)$ \\
\hline Period of tracheal intubation, day, median (range) & 58 & $(24-109)$ \\
\hline CLD, $\mathrm{n}(\%)$ & 23 & $(100)$ \\
\hline PDA, $\mathrm{n}(\%)$ & 16 & $(70)$ \\
\hline NEC, $\mathrm{n}(\%)$ & 3 & $(13)$ \\
\hline IVH, $\mathrm{n}(\%)$ & 2 & $(9)$ \\
\hline PVL, $\mathrm{n}(\%)$ & 0 & $(0)$ \\
\hline Death before discharge, $\mathrm{n}(\%)$ & 0 & $(0)$ \\
\hline
\end{tabular}

Table 2

Characteristics of VAP episodes $(n=34)$

\begin{tabular}{|lll|}
\hline Variables & & \\
\hline Age of onset, day, median (range) & 29.5 & $(9-73)$ \\
\hline Period of tracheal intubation before onset, day, median (range) & 29 & $(3-69)$ \\
\hline
\end{tabular}

\section{Comparison of causative bacteria and surveillance culture}

We initially compared the results of surveillance culture using TA samples collected at VAP onset (causative bacteria) and those of surveillance culture using samples collected before VAP onset. Surveillance culture was performed 1 and 2 weeks before VAP onset in 25 of 34 VAP cases (Fig. 1). Results showed that the concordance rates between causative bacteria and NC 1 and 2 wks were $68 \%$ 
and 44\%, respectively, and those between causative bacteria and TA 1 and 2 wks were $72 \%$ and $60 \%$, respectively (Table 3 ).

Table 3

Concordance rates between pathogenic bacteria and surveillance culture

\begin{tabular}{|lll|}
\hline & Concordance & Concordance rate (\%) \\
\hline Surveillance culture & & \\
\hline NC 1 wk & $17 / 25$ & 68 \\
\hline NC 2 wks & $12 / 25$ & 48 \\
\hline TA 1 wk & $18 / 25$ & 72 \\
\hline TA 2 wks & $16 / 25$ & 64 \\
\hline
\end{tabular}

\section{Concordance rates according the concurrent performance of Gram staining}

In $34 \mathrm{VAP}$ cases, the concordance rate between referable TA and causative bacteria was $53 \%$ (Table 4). The results from 21 referable TAs in 34 cases were consistent with those from TA samples collected at the onset of VAP in terms of color (positive or negative) and shape (bacillus or coccus) (Fig. 1). The referable TAs in 21 cases were subsequently compared with the causative bacteria. Results showed that it had a significantly high concordance rate at $86 \%(p=0.022)$.

Table 4

Concordance rates between pathogenic bacteria and referable TA and/or Gram staining

\begin{tabular}{|llll|}
\hline & Concordance & $\begin{array}{l}\text { Concordance } \\
\text { rate }(\%)\end{array}$ & p \\
\hline Referable TA alone & $19 / 34$ & 56 & \\
\hline Gram staining & $32 / 34$ & 91 & $\begin{array}{l}\mathrm{p}=0.022 \text { vs. Referable TA } \\
\text { alone }\end{array}$ \\
\hline $\begin{array}{l}\text { Referable TA matched with Gram } \\
\text { staining }\end{array}$ & $18 / 21$ & 86 & \\
\hline NC 1 wk (2 wks): A nasal cavity collected 1-7 days (8-14 days) before the onset
\end{tabular}

TA 1 wk (2 wks): A tracheal aspiration collected 1-7 days (8-14 days) before the onset

Referable TA: The result of surveillance culture 4-10 days before the onset

Gram staining: A Gram staining of a tracheal aspiration collected at the onset

\section{Assessment of causative bacteria that are challenging to treat with commonly used antibiotics}


The characteristics of methicillin-resistant Staphylococcus aureus (MRSA) and Pseudomonas aeruginosa, which are challenging to treat with commonly used antibiotics, are shown in Table 5. The two causative bacteria and referable TA were compared. Results showed that the sensitivity and specificity in identifying these bacteria were $50 \%$ and $100 \%$, respectively.

Table 5

Examination of pathogenic bacteria that is difficult to treat with usual antibiotics (MRSA or Pseudmonas aeruginosa)

\begin{tabular}{|lllll|}
\hline & & \multicolumn{3}{l|}{ In tracheal aspiration collected at the onset } \\
\hline \multirow{3}{*}{ In available TA } & Detected & Not detected & Total \\
\cline { 2 - 5 } & Detected & 7 & 0 & 7 \\
\cline { 2 - 5 } & Not detected & 7 & 20 & 27 \\
\cline { 2 - 5 } & Total & 14 & 20 & 34 \\
\hline
\end{tabular}

\section{Discussion}

This study first showed the efficacy of surveillance culture in detecting the causative bacteria of VAP in neonates. In adult patients, surveillance culture is considered important [2]. In several studies on adult VAP cases in the intensive care unit (ICU), surveillance culture of TA samples was found to be effective in identifying causative bacteria, with a concordance rate of up to $35-85 \%$ [2], and the efficacy of this method is still investigated.

Michel et al. reported that the sensitivity of identifying causative bacteria using TA samples every 72$96 \mathrm{~h}$ was 83\% [5]. According to a systematic review by Brusselaer et al., the sensitivity and specificity of surveillance culture using lower respiratory tract specimens were $75 \%$ and $92 \%$, respectively. Hence, it has a high accuracy in assessing the causative bacteria of VAP [6]. Conversely, Carlos et al. compared two groups receiving VAP treatment: one treated according to the results of surveillance culture using TA samples and the other treated according to treatment guidelines [7]. The treatment efficacy rates were $77.4 \%$ in the surveillance culture group and $97.9 \%$ in the guideline treatment group. These results showed that whether surveillance culture is effective in evaluating the causative bacteria of adult VAP is controversial. Ichikawa et al. have reported the efficacy of surveillance culture for late-onset bacterial infection among neonates [8]. However, of 23 episodes, only three involved pneumonia, and the efficiency of surveillance culture in VAP was unclear.

Based on the concordance rate of surveillance culture and causative bacteria in our study, when the collection time of specimens was closer to the onset of VAP, the concordance rate was higher.

Furthermore, tracheal secretion was more likely to match with the causative bacteria than the NC culture. However, neither of the two had significant differences. Regarding the appropriate sample collection time, Delclaux et al. have reported that in 16 of 24 VAP adult cases, the same causative bacteria produced colony in tracheal tubes 2-6 days prior to the event [9]. Based on meta-analysis results of adult VAP 
cases, surveillance culture performed close to the onset of VAP had higher sensitivity and specificity in identifying the causative bacteria of VAP than surveillance culture performed earlier. Hence, when the samples were collected close to the onset, the concordance rate might be higher [6]. However, Boots et al. have shown that even surveillance culture using TA samples collected a day before the onset of VAP could yield negative results; therefore, samples should be collected at the time of onset [10].

In our hospital, bacterial culture results are available 4 days after sample submission. The concordance rate of clinically available TA from 4-10 days prior to the onset of VAP and the causative bacteria of VAP was $56 \%$. This indicates a high rate of failure in the evaluation of causative bacteria based on surveillance culture alone. Katayama et al. have reported that Gram staining at the onset of VAP was effective in identifying the causative bacteria of VAP in the NICU [3]. In our study, the concordance rate of Gram staining and the causative bacteria was as high as $91 \%$, thereby confirming the efficacy of the additional use of Gram staining. Notably, Gram staining does not reveal the bacterial species, even if it is positive or negative or a bacillus or coccus. Moreover, it cannot identify whether it is multidrug resistant. Thus, concurrently performing surveillance culture and Gram staining can lead to a more precise identification of the causative bacteria. Therefore, we assessed the causative bacteria using combined Gram staining and surveillance culture of TA collected on the day of VAP onset. We considered surveillance culture as an indicator of causative bacteria identification only if the color of the stained bacteria (positive or negative) and the shape (bacillus or coccus) were similar those in the surveillance culture collected 4-10 days prior to the onset of VAP. This shows the advantage of using an early approach against drug-resistant bacteria.

In our hospital, MRSA and Pseudomonas aeruginosa were the drug-resistant causative bacteria of VAP. Similarly, the sensitivity and specificity of surveillance culture in VAP caused by MRSA in the adult ICU were $70 \%$ and $92 \%$, respectively. This result indicated that surveillance culture is effective [11]. If these resistant bacteria are detected on surveillance culture, broad-spectrum antibiotics should be considered.

The current study had some limitations. First, the small sample size was small, and it had a retrospective design. Second, the conditions of resistant bacteria could differ between facilities; therefore, data on MRSA and Pseudomonas aeruginosa could be influenced by different conditions.

The concurrent use of surveillance culture and Gram staining may be effective in identifying causative bacteria in VAP. However, prospective, randomized studies on the outcome of initial antibiotic therapy, rather than broad-spectrum antibiotics, based on the result of both surveillance culture and Gram staining should be conducted to provide more insight regarding the appropriate treatment for VAP in neonates.

\section{Abbreviations}

NICU, neonatal intensive care unit

VAP, ventilator-associated pneumonia 
NC, nasal cavity

TA, tracheal aspirate

\section{Declarations}

Ethics approval and consent to participate: This study adheres to the ethical principles of the Declaration of Helsinki. It was approved by the Ethics Committee of Takatsuki General Hospital. Informed consent was obtained from the patient's parent.

Consent for publication: Not Applicable

Availability of data and material: The datasets used and/or analyzed during the current study are available from the corresponding author on reasonable request.

Competing interests: The authors declare that they have no competing interests.

Funding: This research did not receive any specific grant from funding agencies in the public, commercial, or not-for-profit sectors

Authors' contributions: Y.U. and M.E. wrote the main manuscript text and made figures and tables. M.S. contributed to the study of statistical methods. S.K., A.T., H.I., H.M., and Y.K. contributed to the data collection. All authors reviewed the manuscript.

Acknowledgements: We thank the nursing and medical staff of the NICU of Takatsuki General Hospital and the laboratory staff for providing technical assistance.

\section{References}

1. Srinivasan R, Asselin J, Gildengorin G, Wiener-Kronish J, Flori HR. A prospective study of ventilatorassociated pneumonia in children. Pediatrics. 2009;123:1108-15.

2. Luna CM, Bledel I, Raimondi A. The role of surveillance cultures in guiding ventilator-associated pneumonia therapy. Curr Opin Infect Dis. 2014;27:184-93.

3. Katayama Y, Minami H, Enomoto M, Takano T, Hayashi S, Lee YK. Usefulness of Gram staining of tracheal aspirates in initial therapy for ventilator-associated pneumonia in extremely preterm neonates. J Perinatol. 2010;30:270-4.

4. Carvalho EM, Massarollo PC, Levin AS, Isern MR, Pereira WL, Abdala E, et al. Comparative study of etiological diagnosis of nosocomial pneumonia. Braz J Infect Dis. 2008;12:67-74.

5. Michel F, Franceschini B, Berger P, Arnal JM, Gainnier M, Sainty JM, et al. Early antibiotic treatment for BAL-confirmed ventilator-associated pneumonia: A role for routine endotracheal aspirate cultures. Chest. 2005;127:589-97. 
6. Brusselaers N, Labeau S, Vogelaers D, Blot S. Value of lower respiratory tract surveillance cultures to predict bacterial pathogens in ventilator-associated pneumonia: systematic review and diagnostic test accuracy meta-analysis. Intensive Care Med. 2013;39:365-75.

7. Luna CM, Sarquis S, Niederman MS, Sosa FA, Otaola M, Bailleau N, et al. Is a strategy based on routine endotracheal cultures the best way to prescribe antibiotics in ventilator-associated pneumonia? Chest. 2013;144:63-71.

8. Ichikawa S, Hoshina T, Kinjo T, Araki S, Kusuhara K. Efficacy of periodic surveillance in a neonatal intensive care unit in the presumption of causative pathogens of late-onset bacterial infection. Am J Infect Control. 2017;45:251-4.

9. Delclaux C, Roupie E, Blot F, Brochard L, Lemaire F, Brun-Buisson C. Lower respiratory tract colonization and infection during severe acute respiratory distress syndrome: Incidence and diagnosis. Am J Respir Crit Care Med. 1997;156:1092-8.

10. Boots RJ, Phillips GE, George N, Faoagali JL. Surveillance culture utility and safety using low-volume blind bronchoalveolar lavage in the diagnosis of ventilator-associated pneumonia. Respirology. 2008;13:87-96.

11. Chan JD, Dellit TH, Choudhuri JA, McNamara E, Melius EJ, Evans HL, et al. Active surveillance cultures of methicillin-resistant Staphylococcus aureus as a tool to predict methicillin-resistant S. aureus ventilator-associated pneumonia. Crit Care Med. 2012;40:1437-42.

\section{Figures}

\section{VAP episodes in 23 neonates (Table 2).}

\section{In 25 episodes, surveillance culture was performed (Table 3).}

\section{Figure 1}

Ventilator-associated pneumonia episodes for each analysis 